\title{
AVALIAÇÃO DE ATEROMA EM ARTÉRIA CARÓTIDA ATRAVÉS DE RADIOGRAFIAS PANORÂMICAS
}

\author{
EVALUATION OF ATEROMA IN CAROTIDE ARTERY THROUGH PANORAMIC RADIOGRAPHS
}

TORQUATO, Patricia Baia1 ${ }^{1}$ ROSENDO, Stallone Augusto Rodrigues ${ }^{1}$, SOUSA, André Luca Araujo de ${ }^{2 *}$, FIRMINO, Brunna da Silva ${ }^{2}$, PINTO, Antonione Santos Bezerra ${ }^{2,3}$, ARAÚJO, Thyago Campos Leite de ${ }^{1}$

${ }^{1}$ Centro Universitário Doutor Leão Sampaio. Juazeiro do Norte - CE, Brasil.
${ }^{2}$ Universidade Estadual do Piauí. Parnaíba - PI, Brasil.
${ }^{3}$ Instituto de Educação Superior do Vale do Parnaíba. Parnaíba - PI, Brasil.

\begin{abstract}
RESUMO
A formação do ateroma (placas ateromatosas calcificadas) é o resultado de um acúmulo de moléculas de gordura na forma de lipídeos envoltos por um tecido fibroso aderido as paredes arteriais. As calcificações na artéria carótida podem ser observadas em radiografias panorâmicas. Foram avaliadas radiografias panorâmicas no Centro de Imaginologia do Centro Universitário Doutor Leão Sampaio com intuito de verificar a prevalência de imagens sugestivas de placas aterosclerósticas calcificadas. Das 2627 radiografias panorâmicas analisadas, cinco apresentaram sugestão de ateroma na bifurcação da artéria carótida, tendo a prevalência nas radiografias de pacientes entre 41 a 71 anos e com uma predominância de calcificações do lado direito. Por meio destas radiografias o cirurgião-dentista pode precocemente identificar essas possíveis manifestações patológicas e encaminhar o paciente para o atendimento médico especializado. Desta maneira prevenindo para a progressão de doenças graves que podem levar o paciente a distintos estados de invalidez ou a morte.
\end{abstract}

Descritores: Radiografia Panorâmica, Artérias Carótidas, Calcificação Vascular.

\begin{abstract}
The formation of atheroma (calcified atheromatous plaques) is the result of an accumulation of fat molecules in the form of lipids surrounded by fibrous tissue adhering to the arterial walls. Carotid artery calcifications can be noticed on panoramic radiographs. Panoramic radiographs were evaluated at the Imaging Center of the Doctor Leão Sampaio University Center in order to verify the prevalence of suggestive images of calcified atherosclerostic plaques. Of the 2627 panoramic radiographs analyzed, five showed suggestion of atheroma at the carotid artery bifurcation with the prevalence on radiographs of patients between 41 and 71 years and with a predominance of right side calcifications. Through these $x$-rays the dentist can identify early these possible pathological manifestations and refer the patient to specialized medical care. Thus preventing the progression of serious diseases that may lead the patient to different states of disability or death.
\end{abstract}

Descriptors: Radiography Panoramic, Carotid Arteries, Vascular Calcification.

\section{INTRODUÇÃO}

A formação do ateroma (placas ateromatosas calcificadas) é o resultado de um acúmulo de moléculas de gordura na forma de lipídeos envoltos por um tecido fibroso aderido as paredes arteriais ${ }^{1}$.

As artérias carótidas são responsáveis pela irrigação cerebral. Quando obstruídas por placas ateroscleróticas, podem ocasionar manifestações de isquemia cerebral desencadeando o Acidente Vascular Cerebral (AVC), que pode se apresentar de duas maneiras, o hemorrágico resultante do rompimento dos vasos sanguíneos, sobretudo da artéria carótida e, o tromboembólico decorrente de uma obstrução na bifurcação da artéria carótida ${ }^{2}$.

Os fatores de risco para o desenvolvimento de ateromas englobam: hábitos deletérios tais como tabagismo e etilismo, sedentarismo, idade avançada, hipertensão arterial, diabetes mellitus, sedentarismo, e triglicérides no sangue ${ }^{3}$.

O emprego de exames por imagens na Odontologia estabelece um importante instrumento que auxilia no diagnóstico e planejamento terapêutico dos processos patológicos dos dentes e dos ossos da face. Dentre os exames por imagem, destaca-se a radiografia panorâmica por ser um exame radiográfico abrangente e de simples 
execução, com capacidade de reproduzir em um único filme todos os elementos dentários, suas estruturas de suporte, além dos limites ósseos das fossas nasais, seios maxilares e articulação temporomandibular (ATM). É também capaz de evidenciar achados que ao exame clínico não seriam revelados, comprometendo assim, o prognóstico de um tratamento ${ }^{4,5}$.

As calcificações na artéria carótida podem ser observadas em radiografia panorâmica dependendo do grau em que se encontram, as placas ateromatosas calcificadas podem ser visualizadas nas laterais da radiografia panorâmica na altura da junção intervertebral C3 e C4 distinguindo das estruturas radiopacas dessa região ${ }^{3}$

Sendo assim, o objetivo do presente estudo é avaliar a prevalência da detecção de placas ateroscleróticas calcificadas através de radiografias panorâmicas, associando com gênero, idade e o lado acometido.

\section{MATERIAL E MÉTODOS}

O presente estudo caracteriza-se como uma pesquisa transversal com caráter retrospectivo e compõe-se da observação de radiografias panorâmicas realizadas em um aparelho modelo HF 100 EAGLE (Dabi Atlante), no período de janeiro de 2013 a setembro de 2016, no laboratório de Imaginologia odontológica de uma instituição de ensino superior privada de odontologia na cidade de Juazeiro do Norte - CE, Brasil.

Todos os procedimentos deste estudo seguiram os princípios éticos estabelecidos pela legislação em vigor, deste modo foi submetido ao Comitê de ética em Pesquisa (CEP) do Instituto Leão Sampaio de Ensino Universitário, conforme a resolução CNS 466/2012, e aprovado sob o CAAE número 54002316.1.0000.5048. Foram selecionadas, como amostra de conveniência, 2627 radiografias panorâmicas.

Os critérios para seleção das amostras foram radiografias de pacientes com idade a partir de 18 anos e ambos os gêneros e radiografias panorâmicas apresentando bom padrão técnico para interpretação, ou seja, sem distorções, com contraste e densidade médios. Logo, foram excluídos da pesquisa radiografias de pacientes com idade inferior a 18 anos, radiografias que não possuíam dados referentes a idade, gênero e imagens com baixa qualidade para diagnóstico, ou seja, imagens mal armazenadas e processadas.

As avaliações das radiografias panorâmicas foram realizadas por acadêmicos previamente treinados e por profissionais da área da Radiologia odontológica. Após as radiografias serem inspecionadas em um ambiente com baixa luminosidade, armazenadas em um computador da marca Samsung e processador Cori i3, foram abertas no programa panorâmico DABI EAGLE, com lente de ampliação de até 16 vezes. Houve a análise da região abaixo da mandíbula em ambos os lados e estruturas adjacentes. A fim de detectar a presença ou não de ateroma em artérias carótidas. Os dados coletados foram registrados em uma ficha específica, e os resultados obtidos foram tabulados no programa Excel para análise através da estatística descritiva.

\section{RESULTADOS}

Das 2627 radiografias panorâmicas analisadas, 06 $(0,23 \%)$ apresentaram sugestão de ateroma na bifurcação da artéria carótida, sendo uma radiografia sugestiva excluída por falta de prontuário. Das 05 (0,19\%) radiografias incluídas na amostra, 02 (40\%) são do gênero masculino e 03 (60\%) gênero feminino (Gráfico 1).

Fonte: Elaborada pelo autor.

Panorâmicas analisadas com sugestão de ateromas em bifurcação de artérias carótidas

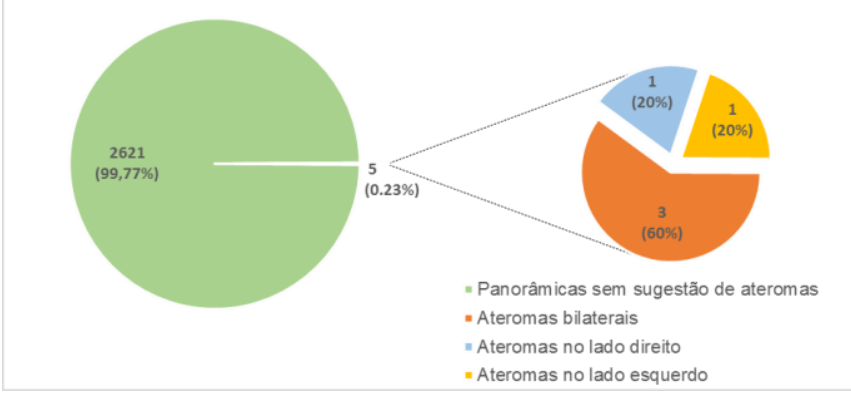

Dentre as radiografias com a sugestão de ateroma nas artérias carótidas, houve prevalência de calcificações em ambos lados com cerca de $60 \%(n=03)$, seguido de radiografias com calcificações do lado direito e esquerdo (Gráfico 1), ambas com 10\% dos casos $(n=1)$ (Figuras 1 e 2).

$E$ as idades dos pacientes das respectivas radiografias eram compreendidas entre 41 a 71 anos.

\section{DISCUSSÃO}

O emprego de exames por imagem na odontologia estabelece uma relevante contribuição no planejamento e diagnóstico dos pacientes. Sendo a radiografia panorâmica um dos exames mais rotineiramente solicitados pelos dentistas, pois dispõe de subsídios efetivos para a maioria de procedimentos feitos na odontologia ${ }^{3}$.

A técnica é de fácil realização, baixo custo, rápida execução, baixa dose de radiação e confortável para o paciente, proporcionando em uma única tomada radiográfica a visualização de dentes, seios maxilares, fossas nasais e estruturas de suporte. Além de permitir a identificação dessas estruturas, radiografias panorâmicas permitem detectar alterações que não venham a ter relação direta com a odontologia ${ }^{6,7}$.

A utilização de radiografias panorâmicas, como auxiliar na detecção de possíveis placas de ateroma que podem vir a causar o Acidente Vascular Cerebral (AVC), ainda é muito debatida na literatura. Contudo, Friedlander \& Lande $(1981)^{8}$ relataram a radiografia panorâmica método válido para a identificação de pacientes com risco para AVC.

A temática em questão não é um assunto totalmente esclarecido, ainda há muitos questionamentos na literatura sobre qual o grau de calcificação na artéria carótida que é capaz de ocasionar a visualização da imagem radiopaca em radiografias panorâmica e qual a vinculação da imagem com o risco clínico de Acidente Vascular Cerebral ${ }^{8}$. 

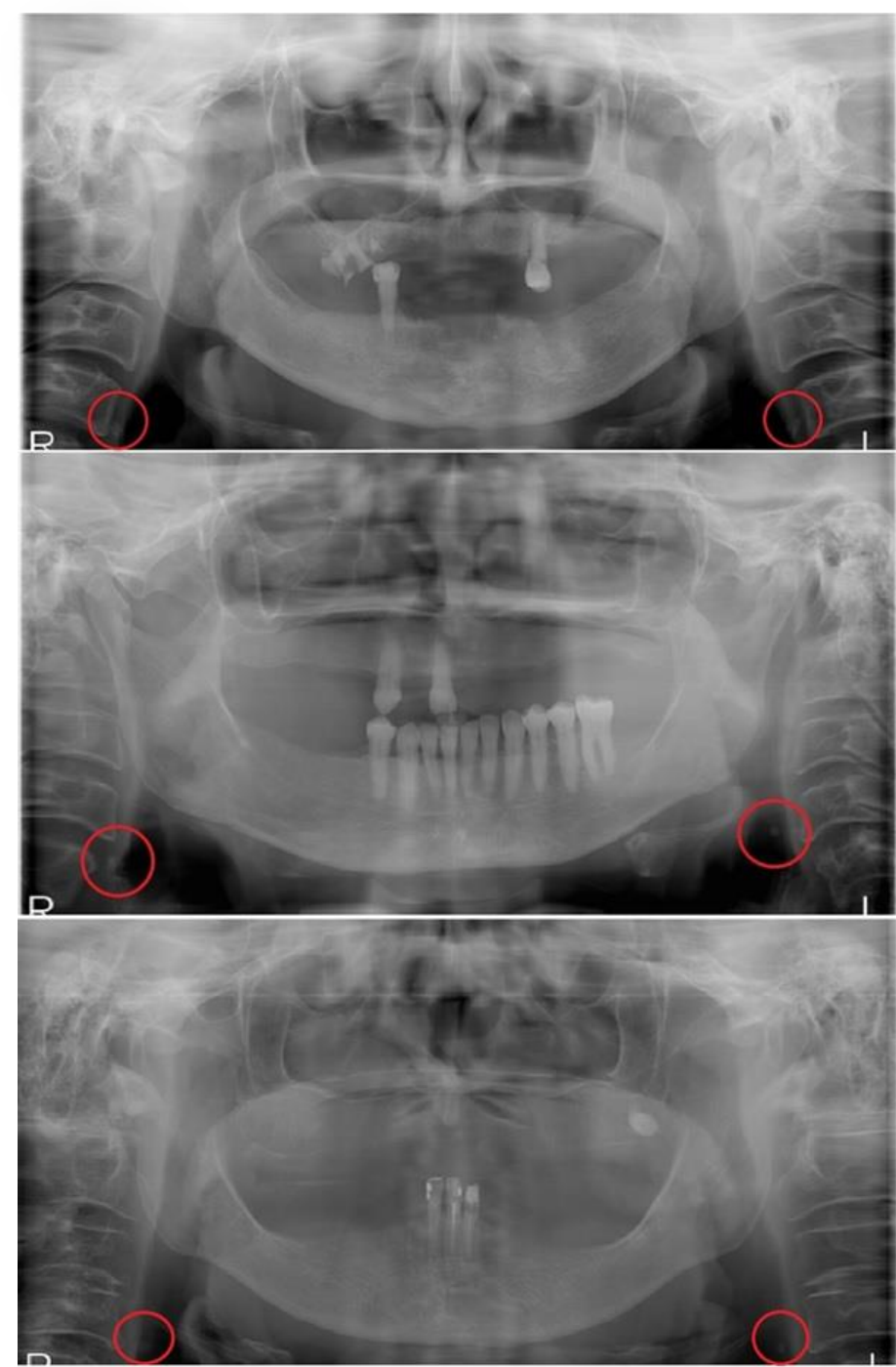

Figura 1. Radiografias panorâmicas com sugestão de ateromas bilaterais nas artérias carótidas

Fonte: Centro universitário Doutro Leão Sampaio, 2016.

Segundo Soares et al. $(2015)^{10}$, a identificação das placas calcificadas de material fibroso na região carotídea pelo dentista é de extrema importância, pois a radiografia panorâmica é um dos exames mais utilizado para auxílio diagnóstico, plano de tratamento e pode auxiliar no diagnóstico precoce de uma patologia mais grave.

De acordo com o presente estudo, a partir das 2627 radiografias panorâmicas analisadas, observou-se uma provável calcificação da placa de ateroma em 05 (0,19\%) radiografias de pacientes com as idades entre 41 e 71 anos. Entre as radiografias com esse tipo de alteração, 02 $(40 \%)$ são do gênero masculino e $03(60 \%)$ do gênero feminino, assemelhando-se com o estudo realizado por Carter et al. $(1997)^{11}$, onde foram avaliadas 1175 radiografias panorâmicas sendo observado a presença de ateroma em artéria carótida em 23 mulheres e 23 homens. Contudo, Obha et al. (2003) ${ }^{12}$ realizou uma observação em pacientes com idade acima de 80 anos para verificar a presença de ateromas da artéria carótida através de radiografias panorâmicas como método de diagnóstico. Das 659 radiografias analisadas, $33(5 \%)$ apresentavam calcificação da artéria carótida. Entre estes achados 8 eram homens e 25 mulheres, divergindo da presente pesquisa, onde não teve prevalência entre os gêneros.

Tunas et al. (2012) ${ }^{13}$ realizaram uma pesquisa para avaliar a presença de placa de ateromas em radiografias panorâmica da Clínica Odontológica de Radiologia da Universidade Estácio de Sá (Unesa), Rio de Janeiro. Os critérios de seleção dos prontuários foram ter radiografia panorâmica e pacientes com mais de 50 anos. Dos 300 prontuários, $29(9,6 \%)$ atendiam aos dois critérios. A avaliação das radiografias panorâmicas foi feita por dois examinadores que, em caso de dúvida, chegaram a um consenso. Dos 29 pacientes examinados, seis $(20,68 \%)$ apresentaram ateromas de carótida. Estes pacientes tinham idade compreendida entre 52 e 73 anos, assemelhando as idades dos pacientes do presente estudo.

Os resultados obtidos por Obha et al. $(2003)^{12}$ apontaram também um predomínio de calcificações no lado direito em $74 \%$ dos casos, assemelhando-se ao presente estudo, onde das 05 radiografias com ateromas, em 04 haviam calcificações na artéria carótida do lado direito (Figuras 1 e 2).
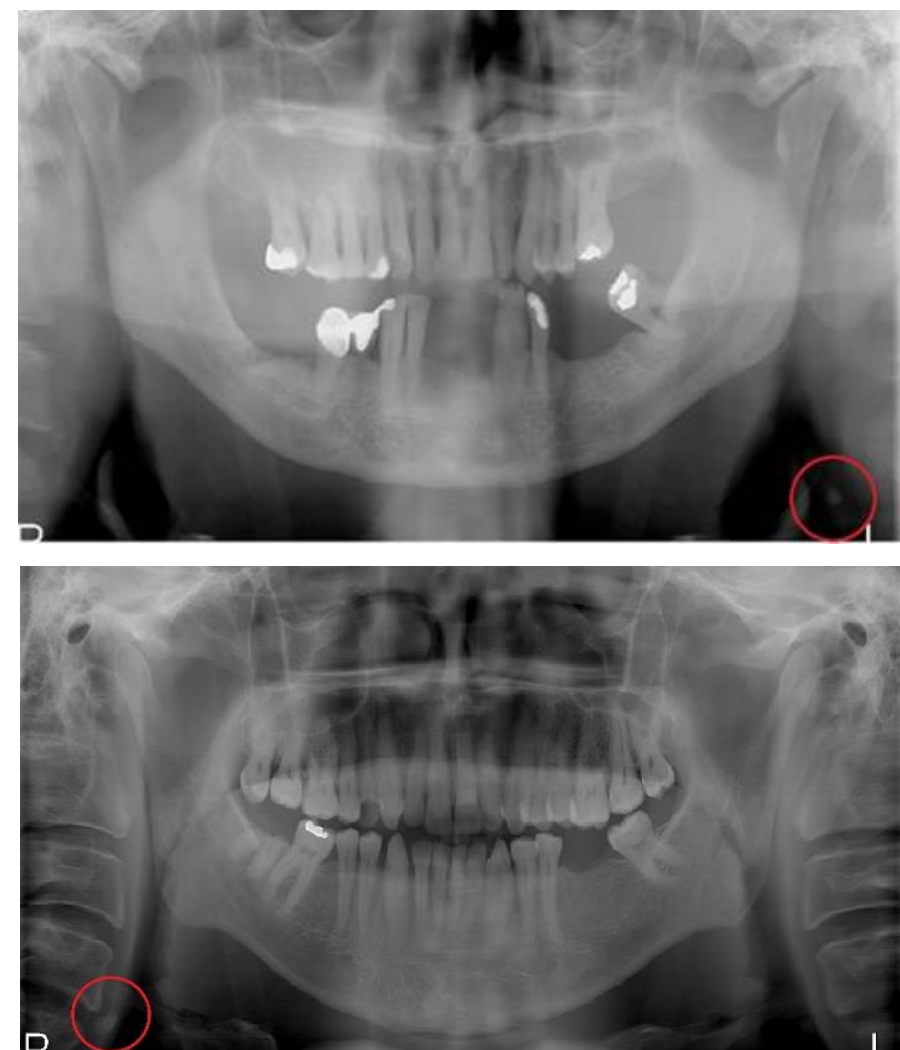

Figura 2. Radiografias panorâmicas com sugestão de ateromas em artéria carótida do lado esquerdo e direito, respectivamente. Fonte: Centro universitário Doutro Leão Sampaio, 2016.

Segundo Friedlander et al. (2005) ${ }^{14}$, a inter-relação entre a calcificação, aterosclerose e 0 avanço e ocorrências clinicas é indefinido, porém Chicano et al. $(2006)^{15}$ relataram em sua pesquisa haver uma interdependência entre a calcificação da carótida e a evolução do AVC. E foi evidenciado de maneira concreta que por meio de radiográficas panorâmicas há o auxílio 
para detecção precoce do risco de AVCs, mas também, o risco de insuficiência cardíaca.

Quando observado as placas ateromatosas na região da bifurcação da artéria carótida, a característica inicial a ser vista é o tamanho da obstrução ${ }^{16}$. Contudo, não é possível exclusivamente com a radiografia panorâmica saber a extensão e o grau de obliteração causado pela placa ateromatosa ${ }^{17}$. Segundo Junior et al. $(2014)^{17}$, a radiografia panorâmica apresenta moderada precisão, alta especificidade e baixa sensibilidade, quando utilizada como complemento no diagnóstico das calcificações ateromatosa.

As artérias carótidas comuns direita e esquerda são as principais artérias da cabeça e pescoço, bifurcando-se em externa e interna. O local dessa bifurcação muda sucintamente e pode, de maneira atípica, ocorrer inferior ao seu nível comum, ausentando-se da área de observação da radiografia panorâmica. Logo, esse exame quando empregado como exame complementar pode não ser eficiente no diagnóstico do paciente com risco de sofrer AVC ${ }^{15,18}$

As placas ateromatosas quando observadas em radiografia panorâmica podem apresentar-se como massas nodulares radiopacas (único ou múltiplos) na altura do espaço intervertebral das vertebras cervicais C3 e C4, abaixo do ângulo da mandíbula, em um ângulo aproximado de 45 graus, adjacentes ao osso hioide ${ }^{19,20}$. É necessária atenção ao diagnóstico diferencial com outras estruturas radiopacas anatômicas e patológicas que podem ser encontrados nessa mesma região ${ }^{10}$ (Figuras 1 e 2).

O diagnóstico diferencial das imagens de ateroma em artérias carótidas em radiografia panorâmica deve ser feito considerando uma série de estruturas anatômicas na cervical e regiões adjacentes e estruturas patológicas tais como: o osso hioide, processo estilóide, cartilagens tritíceas, cartilagem tireóidea, epiglote, calcificações no processo estilóide, ligamentos estilomandibular, palato mole, língua, lóbulo da orelha, tubérculo anterior do atlas e vertebras $^{14,16,18,21}$

Dentre as patologias que podem ser encontradas na mesma região dos ateromas, estão: flebólitos, sialolitos nas glândulas salivares submandibulares, linfonodos calcificados, acne calcificada, tonsilólitos ${ }^{8,11,22}$.

Atualmente, há ainda autores que propõem outras formas de exames radiográficos complementares aplicado por cirurgiões dentistas, de modo a compensar as limitações da radiografia panorâmica, como por exemplo a técnica extra bucal ântero-posterior para comprovar a localização das calcificações ou ainda a teleradiografia lateral| ${ }^{16,22}$.

De acordo com Eid $(2009)^{23}$, apesar da radiografia panorâmica identificar a presença das calcificações sugestivas de ateromas que podem desencadear um AVC, esse exame sozinho não possibilita verificar o grau de obstrução e a correta localização do ateroma, fazendo-se necessário o uso de outros recursos para se obter um diagnóstico definitivo.

Para um diagnóstico preciso das placas ateromatosas pode ser empregado diversos exames como angiografia, Doppler colorido, também chamado Duplex Scan ou Fluxometria Laser Doppler ${ }^{9}$. A Ultrassonografia Doppler é considerada padrão ouro, pois é precisa, rápida e indolor, além de não utilizar radiação ionizante. Os resultados alcançados com esse método produzem imagens que pode ser comparada com a da angiografia. Quando assemelhada a angiografia, a Ultrassonografia produz melhor vantagem por não ser um exame invasivo ${ }^{24,25}$.

A propensão de estudos de exames odontológicos por imagem para diagnóstico de ateroma, não sugerem a substituição dos exames acima descritos, que apresentam elevado grau de confiabilidade, mas se agregam, procurando assim evitar maiores danos ao paciente, a partir de doenças encontradas de maneira eventual ${ }^{10}$. Inúmeros estudos têm evidenciado ligações consistentes de fatores de risco para formação de placas ateromatosas na artéria carótida. Sendo assim, o cirurgião dentista, em poder do histórico de saúde do paciente pode valer-se das informações, auxiliando no diagnóstico diferencial ${ }^{26}$.

Assim, os cirurgiões-dentistas devem encaminhar todos os pacientes com ateromas detectável na radiograficamente para um médico, para confirmação diagnóstica e determinação da magnitude da doença9 .

\section{CONCLUSÃO}

O acometimento de ateroma de placa calcificado em artérias carótidas caracteriza-se como fator de risco para o AVC. E os ateromas de placa podem ser observadas através radiografias panorâmicas como placas radiopacas entre as vertebras C3 e C4 abaixo ou acima do osso hioide cerca de 2 a $4 \mathrm{~cm}$ do ângulo da mandíbula.

Assim, pode-se concluir que essas calcificações acometem em maior proporção indivíduos com idades mais avançadas. Mas em relação a gênero e qual artéria carótida mais acometida, não há ainda estudos suficientes para se definir um padrão.

E por meio destas radiografias o cirurgião-dentista pode precocemente identificar essas possíveis manifestações patológicas e encaminhar o paciente para o atendimento médico especializado. Desta maneira prevenindo para a progressão de doenças graves que podem levar o paciente a distintos estados de invalidez, ou ainda a morte.

\section{REFERÊNCIAS}

1. Coccheri S. Asymptomatic carotid stenosis: natural history anda thetapeutic implications. Pathophysiology of haemostasis and $\begin{array}{lll}\text { thrombosis. } & \text { 2003/2004; } & \text { 33(5-6): }\end{array}$ https://doi.org/10.1159/000083817

2. AITA J. Doença carotídea e AVE Revista Feedback Disponível em: http://www.brasilclinicas.com.br/artigos/ler.aspx?artigolD=146 Acesso em: 12/11/2010

3. Albuquerque DF, Menezes AV, Carlos MX, Kurita LM, Capelozza ALA Detection of calcifications of the carotid artery in panoramic radiographs: a review of the morphology and pathology. Clin Pesq Odontol. 2005; 2(2): 129-136.

http://revodonto.bvsalud.org/scielo.php?script=sci_nlinks\&ref=093027\& pid=S1677-3888201000010000500001\&lng=pt

4. Whaites E. Princípios de radiologia odontológica. Elsevier Health Sciences. 2011.

5. Alves N, Deana NF, Garay I. Detection of common carotid artery calcifications on panoramic radiographs: prevalence and reliability. International journal of clinical and experimental medicine. 2014; 7(8): 1931-1939. https://www ncbi.n/m.nih.gov/pmc/articles/PMC4161533/

6. Romiti CBB. Análise de ocorrência de imagens sugestivas de calcificações da artéria carótida em radiografias panorâmicas. Faculdade de medicina da Universidade Federal do Mato Grosso do Sul [dissertação].

https://repositorio.ufms.br:8443/jspui/handle/123456789/136 
7. Garay I, Olate S. Consideraciones actuales em el estúdio imagenológico de las calcificaciones de tejidos blandos em zona de ángulo mandibular. International journal of odontostomatology. 2013; 7(3): 455-464. http://dx.doi.org/10.4067/S0718-381X2013000300019.

8. Friedlander AH, Lande A. Panoramic radiographic identification of carotid arterial plaques. Oral Surgery, Oral Medicine, Oral Pathology. 1981; 52(1): 102-104. https://doi.org/10.1016/0030-4220(81)90181-X

9. Abreu TQ, Brito Filho SB, Sales KFP, Spyrides KS, Oliveira AEF. Radiografia panorâmica como possível método de diagnóstico de pacientes com risco de acidente vascular cerebral: revisão da literatura. Pesquisa Brasileira em Odontopediatria e Clínica Integrada. 2011; 11(4): 607-613. http://www.redalyc.org/articulo.oa?id=63722200023

10. Soares MQS, Castro RC, Santos PSS, Capelozza ALA, Fischer-Bullen IRR. Contribuição da radiografia panorâmica no diagnóstico de calcificação de ateroma de carótida: relato de caso e revisão de literatura. Rev Port Estom, Med Dent e Cir Maxilo. 2015; 56(2): 127-131. DOI: 10.1016/j.rpemd.2015.04.011

11. Carter LLC, Haller AD, Nadarajah V, Calamel AD, Aguirre A. Use of panoramic radiography among an ambulatory dental population to detect patients at risk os stroke. The journal of the American Dental Association. 1997; 128(7): 977-984. https://doi.org/10.14219/jada.archive.1997.0338

12. Ohba T, Takata Y, Ansai T, Morimoto Y, Tanaka T, Kito S et al. Evaluation of calcified carotid artery ateromas detected by panoramic radiograph among 80-year-olds. Oral Surgery, Oral Medicine, oral Pathology, Oral Radiology, and Endodontology. 2003; 96(5): 647-650. https://doi.org/10.1016/j.tripleo.2003.07.001

13. Tunas ITC. Ateromas de carótida nas panorâmicas: como o clínico pode identificar?. Revista Brasileira de Odontologia. 2013; 6992): 203-206. http://dx.doi.org/10.18363/rbo.v69n2.p.203

14. Friedlander $A H$, Garrett NR, Chin EE, Baker JD. Ultrasonographic confirmation of carotid artery ateromas diagnosed via panoramic radiography. The journal Of the American Dental Association. 2005; 136(5): 635-640. https://doi.org/10.14219/jada.archive.2005.0235

15. Chicano RR, Sánchez REO, Castaño FL, Merino CC, López Fm. Panoramic radiograph as a method for detecting calcified ateroma plaques. Review of literature. Med Oral Patol Oral Cir Bucal. 2006; 11: 261-266. https://www.ncbi.n/m.nih.gov/pubmed/16648765

16. Guimarães GO, Guimarães IEO, Nobile D, Calderón JCM, Saddy MS Radiografia panorâmica: identificação de pacientes suscetíveis ao acidente vascular cerebral por meio de detecção de ateromas na bifurcação da carótida. ConScienyiae Saúde. 2005; (4): 97-104. http://www.redalyc.org/articulo.oa?id=92900411
17. Junior VLB, Luna AHB, Sales MAO, Rodrigues TLC, Sarmento PLDFA, Mello Junior CF. Confiabilidade da radiografia panorâmica digital no diagnóstico de calcificações na artéria carótida. Radiologia Brasileira. 2014; 47(1): 28-32. http://www.rb.org.br/detalhe_artigo.asp?id=2498

18. Kamikawa RS, Pereira MF, Fernandes A, Meurer MI. Study Of the localization of radiopacities similar to calcified carotid ateroma by means of panoramic radiography. Oral Surgery, Oral Medicine, Oral Pathology, Oral Radiology, and Endodontology. 2006; 101(3): 374-378. https://doi.org/10.1016/j.tripleo.2005.03.030

19. Guzmán D, Concha X Rudolph A, Gusmán C. Aporte preventivo de acidente vascular encefálico em pacientes sometidos a exámenes radiográficos em la ragión macilofacial. Revista Dental de Chile. 2007; 99(3): 34-31.

20. Klosek SK, Rungruang T. Topography of carotid bifurcation: Considerations for neck examination. Surgical and Radiologic Anatomy. 2008; 30(5): 383-387. https://doi.org/10.1007/s00276-008-0337-2

21. Yoon SJ, Yoon W, Kim OS, Lee JS, Kang BC. Diagnostic accuracy of panoramic radiography in the detection of calcified carotid artery. Dentomaxillofacial Radiology. 2008; 37(2): 104-107. https://doi.org/10.1259/dmfr/86909790

22. Christou P, Leemann B, Schimmel M, Kiliaridis S, Muller F. Carotid artery calcification in ischemic stroke patientis detected in standard dental panoramic radiographs-a preliminar stdy. Advances in medical sciences. 2010; 55(1): 26-31. https://doi.org/10.2478/v10039-010-0022-7

23. Eid LM. Saúde bucal e aterosclerose da carótida. ComCiência. 2009 (109): $0-0$ http://comciencia.scielo.br/scielo.php?script=sci_arttext\&pid=S1519$76542009000500036 \&$ Ing $=p t \& n r m=$ is

24. Sousa CMR. Diagnostic agreement between panoramic radiographs and color Doppler images of carotid atheroma. J Appl Oral Sci. 2009; 17(1):45-8. http://dx.doi.org/10.1590/S1678-77572009000100009

25. Deahl II ST. Panoramic radiography is sensitive and specific for detection of calcified carotid arteries when compared with the screening test of color Doppler ultrasound. J Evid Base Pract. 2012; 12(3):167-168. https://doi.org/10.1016/j.jebdp.2012.06.009

26. Romano-Souza CM, Krejci L, Medeiros FMM, Graciosa-Filho RG, Martins MFF, Guedes VN, Fenyo-Pereira M. Diagnostic agreement between panoramic radiographs and color Doppler imagens of carotid ateroma. Journal of Applied Oral Science. 2009; 17(1): 45-48. http://dx.doi.org/10.1590/S1678-77572009000100009 